\title{
Group 13 precursor structures and their effect on oxide nanocrystal formation
}

\author{
I. G. Nielsen, S. Sommer, B. B. Iversen
}

Center for Materials Crystallography, Department of Chemistry and iNano, Aarhus University, Langelandsgade 140, 8000 Aarhus, Denmark

idani@chem.au.dk

Commonly classical nucleation theory has been used to explain nucleation, but this is now being challenged as atomic scale techniques has been developed to study solutions showing larger clusters before nucleation [1, 2]. Thus, a new theory including these clusters with predictive value is needed. To achieve this, it is essential to investigate the atomic structure of precursors across different elements as well as chemical environments.

In this study the precursors of group 13 metal oxides have been examined. Al, In and Ga form similar oxides and hydroxides such as $\mathrm{M}(\mathrm{OH})_{3}, \mathrm{MOOH}$ and $\mathrm{M}_{2} \mathrm{O}_{3}$ in solvothermal synthesis. The individual systems exhibit complex polymorphism, which can be controlled with different synthesis parameters such as solvent and temperature, however, the actual mechanisms are unknown.

The precursor structures of the group 13 metal oxides have been determined by combining PDF and EXAFS analysis of the three metal nitrates in various solvents. Across element and solvents the structures were determined to be octahedrally coordinated metaloxygen with further structure.[3] For the gallium system variation of $\mathrm{pH}$, anions and concentration were further investigated using PDF analysis revealing the diverse solution chemistry of gallium [4].

Based on the results, the formation mechanisms of the group 13 metal oxides are discussed, for example reason for the production of $\mathrm{AlOOH}$ at most synthesis conditions instead of the desirable $\gamma-\mathrm{Al}_{2} \mathrm{O}_{3}$ phase $[3,5]$.

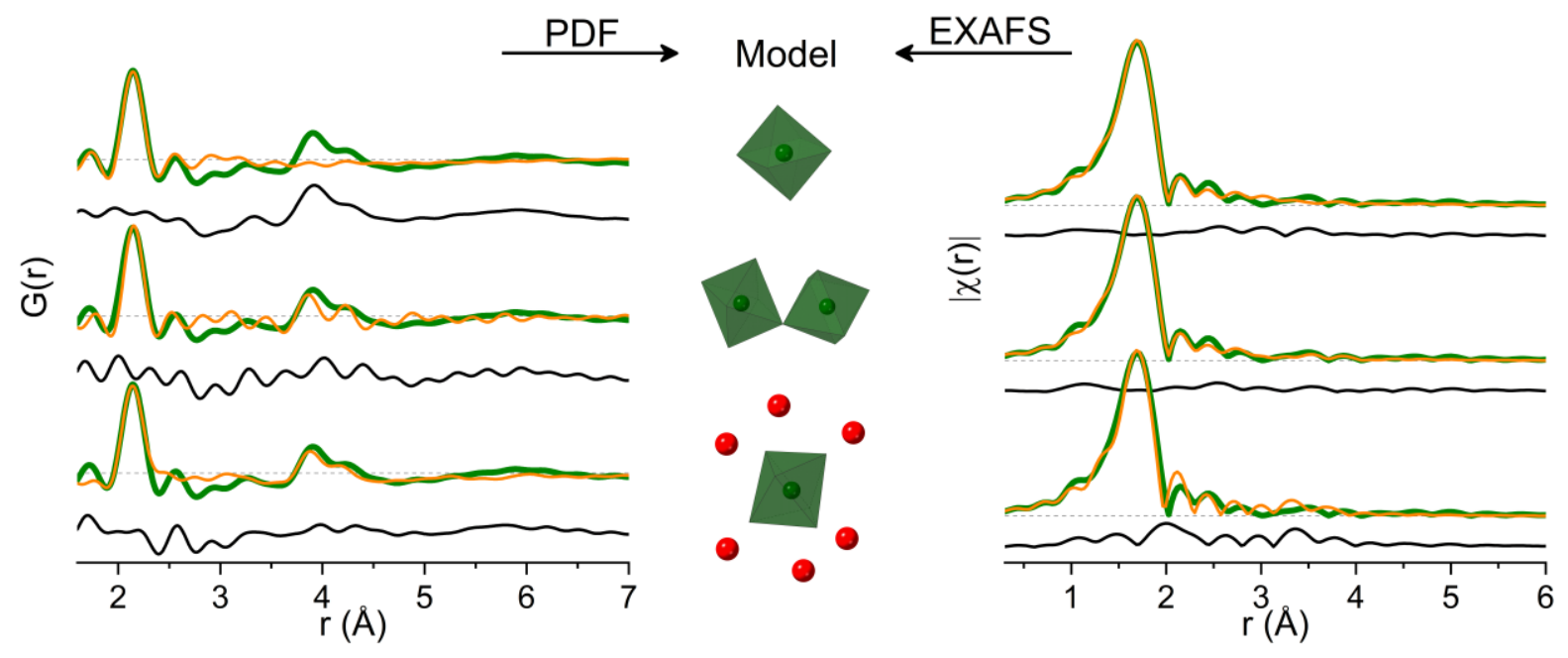

Figure 1. Modelling of both EXAFS and PDF data for the same models.

[1] Bøjesen, E. D. \& Iversen, B. B. (2016). CrystEngComm. 43, 8332-8353

[2] Gebauer, D., Kellermeier, M., Gale, J. D., Bergström, L. \& Cölfen, H. (2014). Chem. Soc. Rev. 43, 2348-2371.

[3] Sommer, S., Nielsen, I. G. \& Iversen, B. B. (2020). Chem. - Eur. J. 26, 1022-1026.

[4] Nielsen, I. G., Sommer, S., Dippel, A.-C. Skibsted, J. \& Iversen, B. B. (2021). Submitted to JACS.

[5] Nielsen, I. G., Sommer, S. \& Iversen, B. B. (2021). Nanoscale 13, 4038-4050.

Keywords: Group 13; precursor structures; EXAFS; PDF; nucleation 